\title{
LASANA -INIA: NUEVA VARIEDAD DE TRIGO HARINERO DE PRIMAVERA PARA LA ZONA CENTRO SUR Y SUR DE CHILE
}

\section{LASANA -INIA: A NEW SPRING BREAD WHEAT CULTIVAR FOR THE CENTRAL SOUTH AND SOUTHERN ZONE OF CHILE}

\author{
Iván Matus ${ }^{1 \mathrm{a} *}$, Ricardo Madariaga ${ }^{1 \mathrm{~b}}$, Claudio Jobet ${ }^{2 \mathrm{a}}$, Javier Zuñiga ${ }^{2 \mathrm{~b}}$ y Christian Alfaro ${ }^{3}$ \\ ${ }^{1 a}$ Instituto de Investigaciones Agropecuarias, Centro Regional de Investigaciones Quilamapu, Casilla \\ 426, Chillán, Chile. \\ https://orcid.org/0000-0003-2161-9286 \\ ${ }^{1 b}$ Instituto de Investigaciones Agropecuarias, Centro Regional de Investigaciones Quilamapu, Casilla \\ 426, Chillán, Chile. \\ https://orcid.org/0000-0002-7222-501X \\ ${ }^{2 a}$ Instituto de Investigaciones Agropecuarias, Centro Regional de Investigaciones Carillanca, Casilla \\ 58-D, Temuco, Chile. \\ https://orcid.org/0000-0003-1756-3523 \\ ${ }^{2 b}$ Instituto de Investigaciones Agropecuarias, Centro Regional de Investigaciones Carillanca, Casilla 58- \\ D, Temuco, Chile. \\ https://orcid.org/ 0000-0002-7267-4472 \\ ${ }^{3}$ Instituto de Investigaciones Agropecuarias, Centro Regional de Investigaciones Rayentue, Casilla 13, \\ Rengo, Chile. \\ https://orcid.org/0000-0003-0861-3349 \\ * Autor para correspondencia E-mail: imatus@inia.cl
}

\section{RESUMEN}

Lasana-INIA es una variedad de trigo harinero (Triticum aestivum L.) de primavera que proviene de un cruzamiento efectuado en el Proyecto de Fitomejoramiento de Trigo del Instituto de Investigaciones Agropecuarias (INIA), en el Centro Regional de Investigación Quilamapu, Chillán, el año 1996. Es una variedad de hábito primaveral, de crecimiento semi-rastrero al estado de plántula. La altura de la planta adulta se considera mediana, y varía entre 85 y $100 \mathrm{~cm}$. La espiga es de color blanco, con barbas largas presentes en toda su extensión. El grano es de color rojo, aspecto vítreo, y forma elíptica. El peso de 1.000 semillas varía entre de 42 a $49 \mathrm{~g}$. Sembrado a mediados de agosto en el Campo Experimental Santa Rosa ( $\left(6^{\circ} 31^{\prime}\right.$ S; $71^{\circ} 54^{\prime}$ O), Chillán, la emisión de espigas ocurre 95 a 100 días después de la siembra. En las localidades de riego, Yungay y Human, 'Lasana-INIA' alcanzó, en promedio, un rendimiento $0,5 \%$ y 3,1\% mayor que la variedad testigo Pandora-INIA. En la localidad de Chillán, en promedio de los cinco años presentó un rendimiento inferior a Pandora-INIA en 4,1\%, pero con diferencias entre años. En suelos de secano húmedo, localidades de Carillanca y Purranque, alcanzó un rendimiento superior en un $9,5 \%$ y $4,2 \%$ respectivamente, respecto de 'Pandora-INIA. 'Lasana-INIA' es un trigo de buen peso del hectolitro y de textura dura. Las cifras de sedimentación de Zeleny (cc), glúten húmedo (\%) y de proteína $(\%)$ lo clasifican como trigo fuerte. Presenta un valor de fuerza de trabajo $\mathrm{W}$ alto, y una buena relación $\mathrm{P} / \mathrm{L}$.

Palabras clave: trigo harinero de primavera, Triticum aestivum L., calidad panadera, rendimiento. 


\section{ABSTRACT}

Lasana-INIA is a spring bread wheat variety (Triticum aestivum L.) originated from a cross carried out in the Wheat Breeding Project of the Agricultural Research Institute (INIA), in the Quilamapu Regional Research Center, Chillán, in 1996. It has a semi-prostrate growth habit in the seedling stage. The adult plant is medium height and varies between 85 and $100 \mathrm{~cm}$. The spike is white with long awns along its full length. The grain is red, vitreous in appearance, and elliptical in shape. The weight of 1,000 seeds varies between 42 to $49 \mathrm{~g}$. It was sown in mid-August in the Santa Rosa Experimental Station $\left(36^{\circ} 31^{\prime}\right.$ S; $71^{\circ} 54^{\prime}$ O), Chillán; head emergence occurred 95 to 100 days after sowing. On average, the yield of Lasana-INIA sown under irrigation in Yungay and Human was $0.5 \%$ and $3.1 \%$ higher than the control variety Pandora-INIA. In Chillán, the average yield over a fiveyear period was $4.1 \%$ lower than Pandora-INIA, but with differences between years. Under rainfed conditions, the yield of Lasana-INIA sown in Carillanca and Purranque was $9.5 \%$ and $4.2 \%$ higher than Pandora-INIA. Lasana-INIA is a bread wheat with a good hectoliter weight and a hard texture. According to its Zeleny sedimentation (cc) value, wet gluten (\%) and protein (\%) contents, it can be classified as strong wheat. It has a high $\mathrm{W}$ value, and a good $\mathrm{P} / \mathrm{L}$ ratio.

Key words: Spring bread wheat, Triticum aestivum L., bread-making quality, yield.

\section{INTRODUCCION}

El trigo en Chile se mantiene como un rubro importante, a pesar del avance de una agricultura diversificada en cultivos y heterogénea en tecnología. Está inserto tanto en la agricultura tradicional como en la de alta tecnología, en la de riego y en la de secano. Es un cereal que contribuye de manera importante a la seguridad alimentaria de Chile, y que involucra a decenas de miles de productores.

Durante los últimos 20 años el trigo se ha sembrado desde la Región Metropolitana (27을

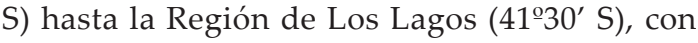
grandes fluctuaciones en el área sembrada y en el rendimiento de grano. $\mathrm{Al}$ respecto, en la temporada 1999-2000 se sembraron 383.527 ha con un rendimiento de $3.8 \mathrm{tha}^{-1}$, en tanto que en la temporada 2019-2020 la superficie descendió a 183.027 ha y en esta misma temporada el rendimiento medio fue de $6.2 \mathrm{t} \mathrm{ha}^{-1}$ (INE, 2019; ODEPA 2020). Se debe considerar que una gran superficie de siembra no siempre es sinónimo de alta producción, debido a los efectos negativos del clima, enfermedades, y a señales de bajos precios originadas en el mercado internacional.

Para predecir lo que vendrá a futuro en este cultivo, se debe tener presente que la superficie sembrada depende principalmente de las expectativas de precio. Las bajas expectativas de buen precio han generado que mucha superficie sembrada en el pasado con este cereal esté hoy día ocupada con cultivos de más alta rentabilidad, como frutales. No obstante, es importante indicar que el trigo está inserto en un sistema productivo, en el que participan cultivos como avena, remolacha, lupino, maíz, papas, raps y cebada, entre otros cultivos, sin considerar aquellos de exportación que pueden competir ventajosamente con el trigo. Además, se debe considerar que la demanda mundial por este cereal está en aumento, y el país debe asegurar un mínimo de producción para su abastecimiento. La cantidad de variedades generadas en el país dependerá de la demanda de los productores y de las exigencias de la industria molinera. En este aspecto, será importante el rol de la economía globalizada y las nuevas tecnologías relativas al manejo del cultivo, el mejoramiento genético, y el uso del grano, todo lo cual puede afectar el intercambio de información técnica con otros países.

Cada nueva variedad que se obtenga debe tener alguna ventaja adicional, como el rendimiento potencial, la calidad industrial del grano, que es cada vez es más importante, a fin de que las variedades desarrolladas en el país, no sean desplazadas por trigos importados, la resistencia genética a las principales enfermedades y plagas, y presentar en lo posible una amplia adaptación a las diferentes zonas agroecológicas productoras de trigo en el país.

En relación a los tipos de variedades sembradas en Chile, aproximadamente del total de superficie sembrada con trigo en el país el $38 \%$ corresponde a siembras con trigos de primavera, y el resto de la superficie se cultiva con variedades de invierno y hábito alternativo.

El objetivo de este trabajo es presentar resultados de la nueva variedad de trigo de primavera, Lasana-INIA, y comparar su comportamiento en cuanto a rendimiento, resistencia a enfermedades, adaptabilidad y calidad industrial, con la variedad de primavera Pandora-INIA, ampliamente sembrada en Chile. 


\section{MATERIALES Y METODOS}

\section{Genealogía}

Lasana-INIA es un cultivar de trigo harinero (Triticum aestivum L.) de primavera que proviene de un cruzamiento de dos líneas experimentales QUI 685-94 y QUP 1707-95, realizado el año 1996, en el Proyecto de Fitomejoramiento de Trigo del Instituto de Investigaciones Agropecuarias (INIA), en el Centro Regional de Investigación Quilamapu, Chillán (36³1 S). La línea experimental QUI 685-94 es un trigo de hábito invernal y la línea QUP 170795 es una línea experimental de hábito primaveral. Las etapas de selección $\mathrm{F}_{2}$ a $\mathrm{F}_{7}$ se realizaron, entre 1998 y 2003, usando el método de pedigrí. El año 2004 se evaluó en un ensayo preliminar de rendimiento, y el año 2005 se incluyó en un ensayo principal de rendimiento. Entre 2006 a 2011 se estudió en ensayos establecidos en diferentes localidades.

\section{Diseño experimental y análisis de datos}

Durante las temporadas 2006-2007 a 2011-2012, se evaluaron ensayos de campo conformados por cultivares y genotipos de trigo de primavera, establecidos en cinco localidades contrastantes en la zona de cultivo de trigo de Chile, ubicada entre los $36^{\circ} 31^{\prime} \mathrm{S} ; 71^{\circ} 54^{\prime} \mathrm{O}$ y $40^{\circ} 86^{\prime} \mathrm{S}$; $73^{\circ} 15^{\prime} \mathrm{O}$.

Los datos se analizaron usando ANOVA y el PROC MIXED de SAS. En el análisis, los genotipos se consideraron de efectos fijos, mientras que el bloque y los bloques incompletos dentro de cada réplica se consideraron como efectos aleatorios. Se calcularon las medias de mínimos cuadrados para los genotipos y la significancia en comparación con el cultivar testigo Pandora-INIA utilizando el comando SAS diff. Se utilizó la variedad de trigo harinero de primavera Pandora-INIA, como testigo, por ser la variedad de primavera más sembrada en el país.

La unidad experimental básica fue una parcela de $2 \times 1 \mathrm{~m}$ con 5 hileras y un espaciado entre hileras de $0,2 \mathrm{~m}$. Para cada ensayo, el diseño experimental básico consistió en 25 genotipos sembrados manualmente con una dosis de siembra de $200 \mathrm{~kg}$ $\mathrm{ha}^{-1} \mathrm{y}$ dispuestos en un diseño de alfa lattice de $5 \times$ $5 \mathrm{~m}$ con cuatro repeticiones.

Se empleó una fertilización estándar para cubrir todas las posibles deficiencias y garantizar que las plantas de trigo siempre recibieran un buen suministro de nutrientes durante todo su ciclo. Las parcelas fueron fertilizadas al momento de la siembra con $47 \mathrm{~kg} \mathrm{ha}^{-1}$ de $\mathrm{N}$ como urea, 120 $\mathrm{kg} \mathrm{ha-1}$ de $\mathrm{P}_{2} \mathrm{O}_{5}, 36 \mathrm{~kg} \mathrm{ha}^{-1}$ de $\mathrm{Mg}, 44 \mathrm{~kg} \mathrm{ha}^{-1}$ de $\mathrm{S}$, $1.05 \mathrm{~kg} h a^{-1}$ de Zn y $1.1 \mathrm{~kg} \mathrm{ha}^{-1}$ de B. Además, se consideraron dos aplicaciones de nitrógeno como urea en las etapas de crecimiento de Zadoks GS 20 (Zadoks et al., 1974) $\left(60 \mathrm{~kg} \mathrm{ha}^{-1}\right)$ y GS $30\left(93 \mathrm{~kg} \mathrm{ha}^{-1}\right)$.
Se utilizó la aplicación de Flufenacet + Flurtamone + Diflufenican $\left(1 \mathrm{~L} \mathrm{ha}^{-1}\right)$ para el control de malezas de preemergencia y una aplicación adicional en GS 29-30 de MCPA (1 L ha $\left.^{-1}\right)+$ Metsulfuron-metil $\left(8 \mathrm{~g} \mathrm{ha}^{-1}\right)$, se consideró como control de postemergencia. Se utilizó un tratamiento de semillas con Mancozeb + Carbendazim para controlar el carbón hediondo (Tilletia foetida) y el carbón volador (Ustilago tritici). No se utilizaron aplicaciones foliares para el control de enfermedades o insectos durante el desarrollo del cultivo.

Se aplicó riego en las localidades de Chillán, Yungay y Human, según demanda del cultivo, para no generar ninguna restricción.

\section{Rendimiento de grano y características agronómicas}

El rendimiento de grano ( $\mathrm{t} \mathrm{ha}^{-1}$ ) se evaluó cosechando con una máquina cosechadora una parcela de $2 \mathrm{~m}^{-2}$. Los días de siembra a espigadura se registraron cuando más del $50 \%$ de las espigas principales dentro de una parcela habían alcanzado GS 55. La altura de la planta $(\mathrm{cm})$ se midió en GS 92 en el centro de la parcela desde la base de la planta y hasta la parte superior de la espiga, excluyendo las aristas.

\section{Calidad del grano}

Las evaluaciones de calidad fueron el índice de dureza $(\%)$, peso del hectolitro $\left(\mathrm{kghL}^{-1}\right)$, porcentaje de proteína $(\mathrm{N} \times 5,7)$, valor de sedimentación $\left(\mathrm{cm}^{3}\right)$ a través del método de Zeleny modificado (Parodi y Wulf, 1996), gluten húmedo (\%), Falling number (seg), Fuerza de Trabajo (W), Tenacidad $(\mathrm{P})$, Extensibilidad $(\mathrm{L})$, Relación $(\mathrm{P} / \mathrm{L})$, de acuerdo con las normas establecidas por el INN (2000) y realizadas en el Laboratorio de Calidad de Trigo de INIA.

\section{Reacción a enfermedades}

Las enfermedades evaluadas fueron roya amarilla (Puccinia striiformis West. f. sp. tritici) y roya colorada de la hoja (Puccinia triticina Erikss.), ó́dio causado por el hongo Blumeria graminis D.C. f. sp. tritici Marchal, y septoriosis de la hoja (Mycosphaerella graminícola (Fuckel) J. Schröt.). La evaluaciones se realizaron en GS 59-60 y 69. La roya de la hoja fue evaluada usando la Escala de Cobb modificada (Peterson et al., 1984), la roya amarilla fue evaluada usando la escala de 0 al 100\%, la que indica el área cubierta por la enfermedad en \% (Kishii et al., 2019), y septoria y oidio usando la Escala modificada de doble digito de Saari y Prescott (1975). En todos los ensayos, localidades y años se evaluaron las cuatro repeticiones de cada genotipo. El valor informado y usado para comparar con la variedad Pandora- 
INIA, para cada enfermedad fue el valor mas alto en cada temporada de cada repetición y cada localidad.

\section{Descripción morfológica, bioquímica y molecular}

La descripción morfológica se realizó durante las temporadas 2006-2007 y 2007-2008, utilizando descriptores morfológicos, exigidos por el sistema chileno de descripción varietal (SAG, 2020).

La caracterización de gluteninas de alto y bajo peso molecular se realizó en base a la metodología establecida por Singh et al. (1991) y Payne et al. (1987). También se evaluó la presencia o ausencia de la traslocación 1RS de Centeno (Koebner, 1995). La dureza del grano se calificó en base a lo establecido por Gautier et al. (1994) y Tranquilli et al. (1999).

\section{RESULTADOS Y DISCUSIÓN}

\section{Descripción morfológica de la planta}

Lasana-INA es una variedad obtenida a partir de un cruzamiento realizado en el programa de mejoramiento genético de trigo de INIAQuilamapu, Chillán. Uno de los progenitores es una línea de hábito invernal y el otro progenitor un trigo de primavera. Ambos progenitores utilizados son a su vez líneas avanzadas seleccionadas también en el mismo programa, a partir de cruzamientos realizados con germoplasma de trigos de primavera provenientes principalmente del Centro Internacional de Mejoramiento Genético de Maíz y Trigo (CIMMYT). Una característica diferenciadora de esta nueva variedad comparada con Pandora-INIA, cultivar de primavera altamente sembrado en Chile, es su hábito de crecimiento semi-rastrero, en estado de plántula (GS15) y macolla (GS30). 'Pandora-INIA' en estos estados de desarrollo tiene un hábito de crecimiento erecto. Esta forma de desarrollo junto con su buena capacidad de macollaje, permite que las plantas cubran rápidamente el suelo, factor que podría contribuir a mejora la competencia con malezas. Su característica de trigo semi-enano y su resistencia a la tendedura, son una gran ventaja, sobre todo en suelos de alto potencial de rendimiento. Sembrado a mediados de agosto en el Campo Experimental Santa Rosa (36 $31^{\prime}$ S; $71^{\circ} 54^{\prime}$ O), Chillán, la emisión de espigas ocurre 95 a 100 días después de la siembra, entre 4 a 5 días más tarde que 'Pandora -INIA' (Mellado et al., 2003). La altura de la planta adulta se considera mediana, y varía entre 85 y $100 \mathrm{~cm}$. La vaina de la hoja bandera presenta fuerte glaucocidad. Las aurículas carecen de antocianina, por lo que presentan color blanco. El tallo es de médula gruesa y es resistente a la tendedura. La espiga es de color blanco, de forma piramidal y laxa a la madurez, aproximadamente de 10 a $12 \mathrm{~cm}$ de largo, presenta glaucocidad media, con barbas largas y presentes en toda su extensión.

El grano es de tamaño intermedio a grande, color rojo, aspecto vítreo, y forma elíptica. El porcentaje en peso de las semillas retenidas en un harnero de mallaje oblongo de $2,3 \mathrm{~mm}$, es de $95 \%$ en promedio; el peso de 1.000 semillas varía entre de 42 a 49 g. Presenta una reacción mediana al fenol.

\section{Rendimiento de grano}

En los ensayos efectuados en las localidades de Yungay y Human, bajo condiciones de riego, 'Lasana-INIA' alcanzó, un rendimiento promedio, mayor en $0,5 \%$ y $3,1 \%$ que la variedad testigo Pandora -INIA, y en la localidad de Chillán, también en suelos regados, en promedio de los seis años presentó un rendimiento inferior a Pandora-INIA en $4,1 \%$, pero con diferencias entre años (Cuadro 1). En las localidades de Carillanca ( $\left.36^{\circ} 69^{\prime} \mathrm{S} ; 72^{\circ} 41^{\prime} \mathrm{S}\right)$ y Purranque $\left(40^{\circ} 86^{\prime}\right.$ S; $\left.73^{\circ} 15^{\prime} \mathrm{O}\right)$, en condición de secano-húmedo, el rendimiento fue $9,5 \%$ y $4,2 \%$, superior a PandoraINIA (Cuadro 1). Este destacado comportamiento en rendimiento en la zona sur es muy relevante, ya que se transforma en una muy buena alternativa para siembras de primavera en esta zona, y con niveles de productividad muy importantes. Se destaca el rendimiento alcanzado el año 2010, en la localidad de Purranque con 13,54 t ha-1, al mismo tiempo en varios años y diferentes localidades esta variedad superó las $10,0 \mathrm{t} \mathrm{ha}^{-1}$, nivel de productividad muy alto considerando que esta es una variedad de hábito primaveral con un ciclo de desarrollo de más o menos 100 días desde siembra a espigadura.

\section{Características fitopatológicas}

La variedad Lasana-INIA durante las 6 temporadas de evaluación ha presentado niveles de ataque a las principales enfermedades que no supera el $10 \%$, y con una reacción de resistencia a moderada resistencia a roya estriada (Puccinia striiformis West. f. sp. tritici) y colorada de la hoja (Puccinia triticina Erikss.) (Cuadro 2), y sin presencia de ataque de oídio causado por el hongo Blumeria graminis D.C. f. sp. tritici Marchal, y tolerancia a septoriosis de la hoja (Mycosphaerella graminícola (Fuckel) J. Schröt.) (Cuadro 3). En comparación con el testigo 'Pandora-INIA', en todos los años de evaluación ha presentado niveles más bajos de roya de la hoja (Cuadro 2), esto es importante ya que sobre $20 \%$ de ataque de esta roya es necesario recurrir a la aplicación de control químico a través de un fungicida foliar. Durante su desarrollo esta variedad no presenta 
Cuadro 1. Rendimiento de grano ( $\left(\mathrm{ha}^{-1}\right)$ de la variedad Lasana-INIA, y del cultivar testigo PandoraINIA, en 3 localidades de riego (R) y 2 localidades de secano (S). Se indican las diferencias respecto de Pandora-INIA.

Table 1. Grain yield ( $\mathrm{ha}^{-1}$ ) of cv. Lasana-INIA compared to $\mathrm{cv}$. Pandora-INIA in three irrigated (R) experimental sites and two rainfed (S) experimental sites. The differences respect to Pandora-INIA are indicated.

\begin{tabular}{|c|c|c|c|c|c|c|}
\hline \multirow[b]{2}{*}{ Localidades } & \multirow[b]{2}{*}{ Año } & \multicolumn{2}{|c|}{ Genotipos } & \multirow[b]{2}{*}{ Diferencia } & \multirow[b]{2}{*}{ Significancia $^{1}$} & \multirow[b]{2}{*}{$\mathrm{CV} \%$} \\
\hline & & $\begin{array}{c}\text { Lasana- } \\
\text { INIA }\end{array}$ & $\begin{array}{l}\text { Pandora- } \\
\text { INIA }\end{array}$ & & & \\
\hline \multirow[t]{7}{*}{ Quilamapu (R) } & 2006 & 10,01 & 10,42 & $-0,41$ & & 4.1 \\
\hline & 2007 & 6,62 & 6,37 & 0,25 & & 6,8 \\
\hline & 2008 & 9,05 & 9,57 & $-0,52$ & $* *$ & 5,7 \\
\hline & 2009 & 9,29 & 9,04 & 0,25 & & 6,2 \\
\hline & 2010 & 9,88 & 11,42 & $-1,54$ & $* *$ & 6,1 \\
\hline & 2011 & 11,42 & 11,87 & $-0,45$ & & 4,2 \\
\hline & Promedio & 9,38 & 9,78 & & & \\
\hline \multirow[t]{7}{*}{ Yungay (R) } & 2006 & 10,16 & 9,65 & 0,51 & $* *$ & 7,3 \\
\hline & 2007 & 7,01 & 7,78 & $-0,77$ & $* *$ & 8,1 \\
\hline & 2008 & 8,76 & 7,92 & 0,84 & $* *$ & 8,3 \\
\hline & 2009 & 10,55 & 11,45 & $-0,9$ & $* *$ & 9,2 \\
\hline & 2010 & 9,85 & 9,95 & $-0,1$ & & 5,5 \\
\hline & 2011 & 8,78 & 8,09 & 0,69 & $* *$ & 6,3 \\
\hline & Promedio & 9,18 & 9,14 & & & \\
\hline \multirow[t]{7}{*}{ Human (R) } & 2006 & 10,37 & 10,29 & 0,08 & & 6,1 \\
\hline & 2007 & 10,64 & 9,42 & 1,22 & $* *$ & 6,2 \\
\hline & 2008 & 9,08 & 9,13 & $-0,05$ & & 9,9 \\
\hline & 2009 & 10,35 & 10,36 & $-0,01$ & & 10,3 \\
\hline & 2010 & 9,42 & 9,32 & 0,1 & & 9,5 \\
\hline & 2011 & 9,26 & 8,78 & 0,48 & $* *$ & 10,4 \\
\hline & Promedio & 9,86 & 9,55 & & & \\
\hline \multirow{7}{*}{ Carillanca (S) } & 2006 & 9,94 & 10,49 & $-0,55$ & & 9,5 \\
\hline & 2007 & 8,00 & 7,93 & 0,07 & & 11,9 \\
\hline & 2008 & 8,38 & 7,97 & 0,41 & $* *$ & 12,2 \\
\hline & 2009 & 7,54 & 6,55 & 0,99 & $* *$ & 9,2 \\
\hline & 2010 & 7,32 & 6,07 & 1,25 & $* *$ & 9,6 \\
\hline & 2011 & 6,98 & 4,99 & 1,99 & $* *$ & 11,2 \\
\hline & Promedio & 8,03 & 7,33 & & & \\
\hline \multirow[t]{7}{*}{ Purranque (S) } & 2006 & 7,69 & 8,19 & $-0,5$ & & 12,1 \\
\hline & 2007 & 10,49 & 10,28 & 0,21 & & 9,3 \\
\hline & 2008 & 8,49 & 6,59 & 1,9 & $* *$ & 11,0 \\
\hline & 2009 & 9,03 & 9,11 & $-0,08$ & & 11,5 \\
\hline & 2010 & 13,55 & 11,38 & 2,17 & $* *$ & 12,1 \\
\hline & 2011 & 8,94 & 10,28 & $-1,34$ & $* *$ & 10,2 \\
\hline & Promedio & 9,69 & 9,31 & & & \\
\hline
\end{tabular}

${ }^{(1)}$ La diferencia es significativa de acuerdo a la prueba de Tukey $\mathrm{P}<0,01$. 
Cuadro 2. Comportamiento a roya estriada ( $P$. striiformis) y roya de la hoja ( $P$. triticina) de la variedad Lasana-INIA, y la variedad testigo Pandora-INIA, en 3 localidades de riego (R) y 2 localidades de secano (S).

Table 2. Stripe rust ( $P$. striiformis) and leaf rust ( $P$. triticina) of cv. Lasana-INIA compared to cv. Pandora-INIA in three irrigated (R) experimental sites and two rainfed (S) experimental sites.

\begin{tabular}{|c|c|c|c|c|c|}
\hline \multirow[b]{2}{*}{ Localidad } & \multirow[b]{2}{*}{ Año } & \multicolumn{2}{|c|}{ Lasana-INIA } & \multicolumn{2}{|c|}{ Pandora-INIA } \\
\hline & & $\begin{array}{c}\text { Roya } \\
\text { estriada }^{(1)}\end{array}$ & $\begin{array}{l}\text { Roya de } \\
\text { la hoja }{ }^{(1)}\end{array}$ & $\begin{array}{c}\text { Roya } \\
\text { estriada }^{(1)}\end{array}$ & $\begin{array}{l}\text { Roya de } \\
\text { la hoja (1) }\end{array}$ \\
\hline \multirow[t]{6}{*}{ Quilamapu (R) } & 2006 & 0 & $5 \mathrm{MS}$ & 0 & $20 \mathrm{MS}$ \\
\hline & 2007 & $5 \mathrm{MS}$ & $5 \mathrm{MS}$ & TMS & $5 \mathrm{MS}$ \\
\hline & 2008 & 5MR & 5MS & TMR & $20 \mathrm{MS}$ \\
\hline & 2009 & 0 & $10 \mathrm{MS}$ & 0 & $40 \mathrm{MS}$ \\
\hline & 2010 & $5 \mathrm{MS}$ & 0 & TMR & 5MS \\
\hline & 2011 & 0 & 0 & 0 & 0 \\
\hline \multirow[t]{6}{*}{ Yungay (R) } & 2006 & 0 & 0 & 0 & 0 \\
\hline & 2007 & 0 & 0 & 0 & 0 \\
\hline & 2008 & 0 & 0 & 0 & 0 \\
\hline & 2009 & 0 & 0 & 0 & 0 \\
\hline & 2010 & 0 & 0 & 0 & 0 \\
\hline & 2011 & 0 & 0 & 0 & 0 \\
\hline \multirow[t]{6}{*}{ Human (R) } & 2006 & $10 \mathrm{MS}$ & 0 & $5 \mathrm{MS}$ & $10 \mathrm{MS}$ \\
\hline & 2007 & 0 & 0 & 0 & $5 \mathrm{MS}$ \\
\hline & 2008 & TMS & 5MS & 0 & $30 \mathrm{MS}$ \\
\hline & 2009 & 0 & $10 \mathrm{MS}$ & 0 & $20 \mathrm{MS}$ \\
\hline & 2010 & $10 \mathrm{MS}$ & 0 & $10 \mathrm{MS}$ & 0 \\
\hline & 2011 & 0 & 0 & 0 & 0 \\
\hline \multirow[t]{6}{*}{ Carillanca (S) } & 2006 & 0 & $\mathrm{TR}$ & 0 & 0 \\
\hline & 2007 & 0 & 0 & 0 & 0 \\
\hline & 2008 & 0 & $10 \mathrm{MR}$ & 0 & 20MS \\
\hline & 2009 & 0 & 0 & 0 & 0 \\
\hline & 2010 & 0 & 0 & 0 & 0 \\
\hline & 2011 & TR & 0 & 0 & 0 \\
\hline \multirow[t]{6}{*}{ Purranque (S) } & 2006 & $10 \mathrm{MR}$ & 0 & $10 \mathrm{MR}$ & 0 \\
\hline & 2007 & 0 & 0 & 0 & 0 \\
\hline & 2008 & 0 & TR & 0 & 0 \\
\hline & 2009 & 0 & 0 & 0 & TMR \\
\hline & 2010 & 0 & 0 & 0 & 0 \\
\hline & 2011 & 0 & 0 & 0 & 0 \\
\hline
\end{tabular}

(1) Valores según Escala de Cobb modificada (Peterson et al., 1984), para roya de la hoja y Kishii et al. (2019), para roya estriada, en la cual la intensidad de ataque puede variar entre 0 y $100 \%$. $(\mathrm{T}=$ trazas), en tanto que la reacción de la planta puede ser: Resistente (R), Moderadamente Resistente (MR), Moderadamente Susceptible (MS), o Susceptible (S).

ningún tipo de clorosis asociada a algún efecto ambiental.

\section{Calidad}

'Lasana-INIA' es un trigo de buen peso del hectolitro y de textura dura. El peso del hectolitro promedio fue de $83,35 \mathrm{~kg} \mathrm{hL}^{-1}$ con un índice de dureza 16,9. El contenido de proteína en el grano está el rango de este tipo de trigos, pero con buenas prácticas de manejo del nitrógeno esta variedad puede alcanzar valores de hasta $12,9 \%$. En sedimentación alcanzó un valor de 45,6, con un rango que vario entre 37,0 y $59,1 \mathrm{~cm}^{3}$ y un valor de gluten humedo fue de $34,2 \%$, con un rango entre 31,0 y $38,4 \%$. Presenta un valor $\mathrm{W}$ alto, en promedio de 327 , pero con un rango que va entre 
Cuadro 3. Comportamiento a oidio (Blumeria graminis) y septoria (Septoria tritici), de la variedad Lasana-INIA, y del cultivar testigo Pandora-INIA, en 3 localidades de riego (R) y 2 localidades de secano (S).

Table 3. Powdery mildew (Blumeria graminis) and septoria leaf blotch (Septoria tritici), of cv. Lasana-INIA compared to cv. Pandora-INIA in three irrigated (R) experimental sites and two rainfed (S) experimental sites.

\begin{tabular}{|c|c|c|c|c|c|}
\hline \multirow[b]{2}{*}{ Localidad } & \multirow[b]{2}{*}{ Año } & \multicolumn{2}{|c|}{ Lasana-INIA } & \multicolumn{2}{|c|}{ Pandora-INIA } \\
\hline & & Oidio $^{(1)}$ & Septoria $^{(1)}$ & Oidio(1) $^{(1)}$ & Septoria $^{(1)}$ \\
\hline \multirow[t]{6}{*}{ Quilamapu (R) } & 2006 & 0 & $3 / 1$ & 0 & $3 / 2$ \\
\hline & 2007 & 0 & 0 & 0 & 0 \\
\hline & 2008 & 0 & 0 & 0 & 0 \\
\hline & 2009 & 0 & $2 / 1$ & 0 & $3 / 2$ \\
\hline & 2010 & 0 & 0 & 0 & 0 \\
\hline & 2011 & 0 & 0 & 0 & 0 \\
\hline \multirow[t]{6}{*}{ Yungay (R) } & 2006 & 0 & 0 & 0 & 0 \\
\hline & 2007 & 0 & 0 & 0 & 0 \\
\hline & 2008 & 0 & 0 & 0 & 0 \\
\hline & 2009 & 0 & 0 & 0 & 0 \\
\hline & 2010 & 0 & 0 & 0 & 0 \\
\hline & 2011 & 0 & 0 & 0 & 0 \\
\hline \multirow[t]{6}{*}{ Human (R) } & 2006 & 0 & 0 & 0 & 0 \\
\hline & 2007 & 0 & 0 & 0 & 0 \\
\hline & 2008 & 0 & 0 & 0 & 0 \\
\hline & 2009 & 0 & 0 & 0 & 0 \\
\hline & 2010 & 0 & 0 & 0 & 0 \\
\hline & 2011 & 0 & 0 & 0 & 0 \\
\hline \multirow[t]{6}{*}{ Carillanca (S) } & 2006 & 0 & $4 / 4$ & 0 & $4 / 5$ \\
\hline & 2007 & 0 & 0 & 0 & 0 \\
\hline & 2008 & 0 & 0 & 0 & 0 \\
\hline & 2009 & 0 & 0 & 0 & 0 \\
\hline & 2010 & 0 & 0 & 0 & 0 \\
\hline & 2011 & 0 & $5 / 5$ & 0 & $5 / 5$ \\
\hline \multirow[t]{6}{*}{ Purranque (S) } & 2006 & 0 & $5 / 5$ & 0 & $6 / 5$ \\
\hline & 2007 & 0 & 0 & 0 & 0 \\
\hline & 2008 & 0 & 0 & 0 & 0 \\
\hline & 2009 & 0 & 0 & 0 & 0 \\
\hline & 2010 & 0 & 0 & 0 & 0 \\
\hline & 2011 & 0 & 0 & 0 & 0 \\
\hline
\end{tabular}

(1) Valores Escala de Saari y Prescott (1975). Escala de 1 a 9.

227 y 487, muy superior a la variedad testigo, y con una buena relación P/L. De acuerdo con sus características de calidad y según la norma chilena se ubica en la categoría de un trigo tipo fuerte a extrafuerte y puede ser considerado un trigo corrector (Cuadro 4).

\section{Caracterización bioquímica y molecular}

La caracterización de las proteínas de alto peso molecular (HMW), indica que 'Lasana-INIA' presenta el alelo 1 en el locus Glu1A, los alelos $17+18$ para el locus Glu1B y 5+10 para el locus
Glu 1D (Payne et al., 1987; Singh et al., 1991). De acuerdo con esta combinación de alelos, y según la escala de 4 a 10 (Payne et al., 1987) alcanza el valor 10, valor máximo que se puede obtener. Es importante indicar que esta variedad no presenta la traslocación 1RS de Centeno (Koebner, 1995). La presencia de esta traslocación produce masas pegajosas, bajo volumen de sedimentación y fermentación deficiente y también una harina de color oscuro (Grayboch, 2001; Zhao et al., 2012; Li et al., 2016).

De acuerdo con la presencia de los alelos Pina- 
Cuadro 4. Características de calidad de la variedad Lasana-INIA, comparada con el cultivar testigo Pandora-INIA.

Table 4. Quality characteristics of cv. Lasana-INIA compared to cv. Pandora-INIA.

\begin{tabular}{lcc}
\hline \multirow{2}{*}{ Características $^{(1)}$} & \multicolumn{2}{c}{ Variedades } \\
\cline { 2 - 3 } & Lasana-INIA & Pandora - INIA \\
\hline Índice de dureza, \% ${ }^{(2)}$ & $16,9(15,9-20,2)^{*}$ & $16,2(14,8-17,3)$ \\
Peso del hectolitro, kg hL ${ }^{-1}$ & $83,35(80.21-86.36)$ & $81,64(79,17-85,00)$ \\
Proteína (N x 5,7), \% & $10,6(9,6-12,9)$ & $10,2(9,1-13,3)$ \\
Sedimentación Zeleny, cm ${ }^{3(3)}$ & $45,6(37,0-59,1)$ & $41,3(35.0-50,5)$ \\
Gluten húmedo, \% ${ }^{(4)}$ & $34,2(31,0-38,4)$ & $35,3(32,5-38,0)$ \\
Falling number (seg) & $427(281-510)$ & $446(208-520)$ \\
Trabajo (W) ${ }^{(6)}$ & $327(227-487)$ & $269(186-332)$ \\
Tenacidad (P) & $108(70-154)$ & $113(69-123)$ \\
Extensibilidad (L) & $114(59-172)$ & $97(49-146)$ \\
Relación (P/L) & $1,2(0,48-2,91)$ & $1,4(0,48-3,44)$ \\
\hline
\end{tabular}

(1) Valores promedio de ensayos efectuados en Chillán, Yungay, Human, Carillanca y Purranque durante los años 2006 a 2011.

(2) Índice de dureza: Valores de 16 a 25 corresponden a un grano duro; valores de 26 a 30 corresponden a un grano semiduro; valores de 31 o más corresponden a un grano blando.

(3) Sedimentación Zeleny: Cifras de 17 a $26,9 \mathrm{~cm}^{3}$ corresponden a un trigo suave; cifras de 27 a $32,9 \mathrm{~cm}^{3}$ corresponden a un trigo intermedio; cifras de 33 a $44 \mathrm{~cm}^{3}$ corresponde a un trigo fuerte; cifras superiores a $44 \mathrm{~cm}^{3}$ corresponde a un trigo muy fuerte.

(4) Gluten húmedo valores igual o mayor a 30\% corresponde a trigo fuerte; entre 29,9 y 25,0\%, corresponde a un trigo intermedio; contenido mínimo de 18,0\% corresponde a un trigo suave.

(5) Falling Number: Bajo 200, trigo con Alfa amilasa activada; entre 200 a 300, trigo con poca cantidad de Alfa amilasa; sobre 300, trigo sin Alfa amilasa.

(6) Valor W (Alveograma): Menor de 160 corresponde a un valor bajo; entre 161 a 250, corresponde a un valor medio; entre 251 a más, corresponde a un valor bueno.

* Rango de valores según localidad.

** Fuente escalas de evaluación: INN. 2000, Laboratorio Farinología del Instituto de Investigaciones Agropecuarias (INIA).

D1b y el Pinb-D1b a partir de Gautier et al. (1994) y Tranquilli et al. (1999), Lasana-INIA se clasifica como un trigo de grano duro.

\section{Zona de cultivo y fechas de siembra}

'Lasana-INIA' se recomienda desde la Región de Nuble hasta la Región de Los Lagos. En suelos de riego la fecha recomendada de siembra es entre mediados de julio y mediados de septiembre. En el secano húmedo de la Región de La Araucanía y Región de Los Ríos y Región de Los Lagos se recomienda desde mediados de agosto hasta finales de septiembre.

\section{CONCLUSIONES}

'Lasana-INIA' es una nueva variedad de trigo harinero de primavera con buen potencial de rendimiento, buen peso hectolitro y textura dura. El grano es de tamaño mediano a grande, rojo y vítreo. De acuerdo con el valor de sedimentación Zeleny, gluten húmedo y proteína se clasifica como un trigo fuerte a extrafuerte. Se puede considerar como un trigo del tipo corrector, cuya harina puede ser utilizada en mezcla para mejorar las características de calidad de otras harinas. Presenta una resistencia moderada a la roya amarilla, la roya de la hoja, al oidio y septoria, lo que es una gran ventaja con respecto a 'Pandora-INIA' y otras variedades ya que no es necesario recurrir a la aplicación de algún fungicida de aplicación foliar.

'Lasana-INIA' se recomienda desde la Región de Ñuble hasta la Región de Los Lagos.

El registro definitivo en el Registro de Variedades Protegidas fue aceptado, el 27 de noviembre del año 2020, por el Servicio Agrícola y Ganadero (SAG) de Chile. 


\section{LITERATURA CITADA}

Gautier, M.F., M.E. Aleman, A. Guirao, D. Marion, and P. Joudrier. 1994. Triticum aestivum puroindolines, two cystine-rich seed proteins: cDNA sequence analysis and developmental gene expression. Plant Molecular Biology 25:43-57.

Graybosch, R.A. 2001. Uneasy unions: Quality effects of rye chromatin transfers to wheat. J. Cereal Sci. 33(1):3-16

INE. 2007. VII Censo Nacional Agropecuario y Forestal. Instituto Nacional de Estadísticas (INE), Santiago, Chile. Disponible en http://www.censoagropecuario.cl/ noticias/08/6/10062008.html. (Consulta 2 diciembre 2020).

INE. 2019. Estadísticas Productivas Agrícolas. Cultivos Anuales Históricos. Trigo. Instituto Nacional de Estadísticas (INE), Santiago, Chile,

INN. 2000. Norma Chilena. Nch 1237-2000. Trigo Harinero- Requisitos. 16 p. Instituto Nacional de Normalización (INE), Santiago, Chile.

Kishii, M., Huerta, J., Tsujimoto, H., and Matsuoka, Y. 2019. Stripe rust resistance in wild wheat Aegilops tauschii Coss.: genetic structure and inheritance in synthetic allohexaploid Triticum wheat lines. Genet. Resour. Crop. Evol. 66 (4): 909-920. https:// doi.org/10.1007/s10722-019-00758-w

Koebner RMD, 1995: Generation of PCR-based markers for the detection of rye chromatin in wheat background. Theor. Appl. Genet. 90:740-745.

Li, Z., T. Ren, B. Yan, F. Tan, M. Yang, and Z. Ren 2016. A mutant with expression deletion of gene Sec-1 in a 1RS.1BL line and its effect on production quality of wheat. PLoS ONE 11(1):e0146943. doi:10.1371/journal. pone. 0146943

Mellado, M., y R. Madariaga. 2003. Pandora INIA, nuevo cultivar de trigo de primavera para Chile. Agricultura Técnica (Chile) 63(3):319-322.
ODEPA. 2020. Estadísticas Productivas Agrícolas. Cultivos Anuales Históricos. Trigo. Oficina de Estudio y Política Agraria (ODEPA), Santiago. Chile. Disponible en: www.odepa. gob.cl (Consulta 5 junio 2020).

Peterson, R., J. Campbell, and A. Hannah. 1984. A diagrammatic scale for estimating rust intensity of leaves and stem of cereals. Can. J. Res. Sect. C 26:496-500.

Parodi, P., y M.H. Wulf. 1996. Expresión de la heterosis en la calidad molinera y panadera de híbridos en trigo. Agricultura Técnica (Chile) 26:97-106.

Payne, P.I., M.A. Nightingale, A.F. Krattiger, and L.M. Holt. 1987. The relationship between HMW glutenin subunit composition and the bread-making quality of British-grown wheat varieties. J. Sci. Food Agric. 40:51-65.

Saari, E., and J. Prescott. 1975. A scale for appraising the foliar intensity of wheat diseases. Plant Dis. Rep. 59:377-380.

SAG. 2020. División Semillas. Servicio Agrícola y Ganadero (SAG), Santiago, Chile. Disponible en: www.sag.gob (Consulta 5 junio 2020).

Singh, N.K., K.W. Shepherd, and G.B. Cornish. 1991. A simplified SDS-PAGE procedure for separating LMW subunits of glutenin. J. Cereal Sci. 14: 203-208.

Tranquilli, G, D. Lijavetzky, G. Muzzi, and J. Dubcovsky. 1999. Genetic and physical characterization of grain texture-related loci in diploid wheat. Molecular and General Genetics 262:846 - 850.

Zadoks, J.C., T.T. Chang, and C.F. Konzak. 1974. A decimal code for the growth stages of cereals. Weed Res. 14, 415-421. https://doi. org/10.1111/j.1365-3180.1974.tb01084.x.

Zhao, C., F. Cui, X. Wang, S. Shan, X Li, Y. Bao, et al. 2012. Effects of 1BL/1RS translocation in wheat on agronomic performance and quality characteristics. Field Crop Res. 127(27):79-84. 